\title{
Wetting of hydrocarbon liquid surfaces by fluorocarbon vapor: a microscopic study
}

\author{
Oleg Gang *, Masafumi Fukuto, Patrick Huber, Peter Pershan
}

Division of Engineering and Applied Science, Harvard University, Cambridge, MA 02138, USA

\begin{abstract}
The wetting of the liquid fluorocarbon (perfluoromethylcyclohexane) on the liquid hydrocarbon (eicosane) surface was studied just above the eicosane melting point, where the eicosane exhibits surface freezing (SF) behavior. The thickness of the wetting layer was controlled by changing the fluorocarbon chemical potential relative to the reservoir through temperature differences $\Delta T$. The evolution of the microscopic structure of the fluorocarbon layer along the normal-to-surface was measured in situ using X-ray reflectivity. Observed effects include suppression of the SF phase for the thinnest absorbed fluorocarbon layer $\left(d \sim 7 \AA\right.$ ) as well as complete wetting that following the $d \sim \Delta T^{-1 / 3}$ law. (C) 2002 Elsevier Science B.V. All rights reserved.
\end{abstract}

Keywords: Wetting; Surface; Fluorocarbon; Hydrocarbon; Alkane; X-ray reflectivity

The physical properties of thin fluid films are important for a variety of technological processes, such as coating, lubrication, and adhesion. They are also the focus of numerous experimental and theoretical studies of wetting phenomena that have contributed significantly to current understanding of statistical physics [1-3]. Liquid surfaces also provide a unique opportunity to study wetting phenomena on a structure less substrate $[4,5]$. Specifically they provide a unique opportunity to study the evolution of the liquid-liquid interface as one of the liquids evolves from a thin 2D wetting layer to the bulk liquid [6].

The hydrocarbon/fluorocarbon system, chosen

\footnotetext{
* Corresponding author. Tel.: + 1-617-495-4015; fax: + 1617-496-4654.

E-mail address: gang@xray.harvard.edu (O. Gang).
}

for this study, is taken from two significantly different classes of organic materials. They typically exhibit mutual phobicity [7] and strong deviations from predictions of solution theory [8]. Nevertheless, both types of molecules are hydrophobic, apolar, with attractive long-range interactions that are purely van der Waals in origin. The specific system that were studied is eicosane $\left(\mathrm{CH}_{3}\left(\mathrm{CH}_{2}\right)_{18} \mathrm{CH}_{3}, \mathrm{C} 20\right)$ /perfluoromethylcyclohexane $\left(\mathrm{PFMC}, \mathrm{C}_{6} \mathrm{~F}_{11} \mathrm{CF}_{3}\right.$ ). Both chemicals were obtained commercially from the Aldrich and Lancaster, respectively, and used as is.

The reasons for choosing these particular molecules are as follows. First, long alkane chains (16-50 carbons) have been shown to demonstrate surface freezing (SF) behavior in the temperature range up to $3{ }^{\circ} \mathrm{C}$, just above their bulk freezing point [9]. In this temperature range the SF phase 
coexists with its own molten bulk. The SF monolayer is formed by surface alkane chains that are oriented nearly normal to the surface. The monolayer has a crystalline structure with hexagonal packing and a correlation length $\sim 1000 \AA$. The lattice constant of the SF phase changes slightly over the range of its existence due to thermal expansion [10]. The vapor pressure of eicosane is negligible in the range of studied temperatures. In contrast PFMC has significant vapor pressure at room temperature $(\sim 0.15$ bar $)$ as well as an electron density that is approximately twice that of eicosane. The high vapor pressure allows good transport of material through the vapor from the reservoir to the substrate while the electron density provides the high contrast for X-ray measurements. Both these facts, together with the PFMC's very low solubility in the long alkanes and low surface tension $\left(12.5 \mathrm{mN} \mathrm{m}^{-1}\right)$, make this system an ideal candidate for study of wetting on molten alkanes.

The experiment had a dual aim. First, to examine if the wetting layer, in any manner, can affect the SF phase. Second, to study the properties of one liquid wetting the surface of another.

The method for creating the thin liquid films is similar to one that was previously used [11]. The schematic view of the chamber and experiment geometry are shown on Fig. 1. The reservoir of liquid PFMC coats the bottom of the chamber. A thick film of PFMC will form spontaneously on a heated liquid (C20) substrate in thermal equi-

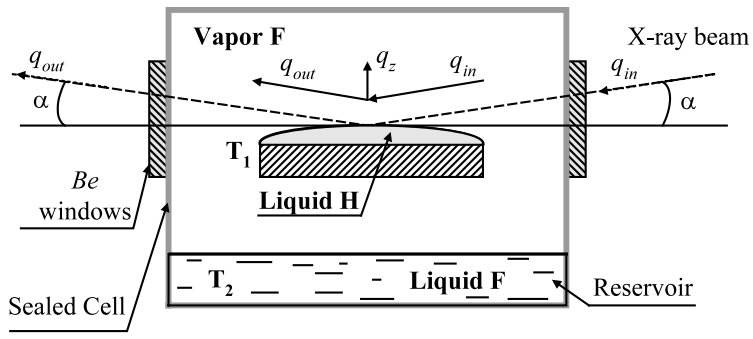

Fig. 1. The schematic view of the X-ray reflectivity experiment. Wetting is controlled by the difference between the temperatures $T_{1}$ of the liquid substrate, eicosane (liquid $\mathrm{H}$ ), and $T_{2}$ of the reservoir, PFMC (liquid F). The normal surface structure is determined from the angular dependence of the X-ray reflectivity. librium with the saturated vapor of a liquid (PFMC) so long as the substrate is above the wetting temperature. The global chemical potential of the PFMC is fixed by the temperature of the liquid reservoir at the bottom of the chamber. The temperature difference $\Delta T$, between the reservoir and adsorbing substrate, causes the chemical potential at the surfaces of the adsorbed liquid to be raised relative that of reservoir by, $\Delta \mu=$ $\left.\frac{\partial \mu_{0}}{\partial T}\right|_{P} \Delta T \approx-S \Delta T$, where $\mu_{0}(P, T)$ is the local, or intrinsic, chemical potential of the liquid, and $S$ is the specific entropy of the liquid. The entropy $S$ is roughly equal to the latent heat of vaporization per PFMC molecule divided by the temperature $T$ [11]. The equilibrium thickness $d$ of the wetting film depends on (1) the attractive van der Waals potential favoring a macroscopically thick film, (2) the difference between the intrinsic chemical potentials of the liquid film at the elevated substrate temperature, $T+\Delta T$, and of the bulk liquid/vapor reservoir at temperature $T$, and (3) the gravitational potential due to the height difference between the sample and the reservoir. The total excess of the free energy per unit area, therefore, can be written as

$\Delta G(d, h, \Delta T)=A_{\mathrm{eff}} /\left(12 \pi d^{2}\right)+d n H \Delta T / T+g h \rho d$,

where $A_{\text {eff }}<0$ is the effective Hamaker constant, $n$ molecular number density, $h$ is height of the film relative to the liquid reservoir and $\rho$ is mass density.

The electron density profile along the normal to the surface of the wetting film was studied by X-ray reflectivity [12] at the Harvard/BNL liquid spectrometer at X22B at NSLS. In this technique the reflected intensity is measured as function of the incident angle $\alpha$ to the surface, while keeping the detector angle at $\beta=\alpha$ (Fig. 1). As mention above, the amount of adsorbed PFMC on liquid substrate was controlled by varying the temperature difference between the molten C20 substrate relative to the PFMC reservoir. Precision measurements required that the thermal homogeneity of the substrate $(\sim 50 \mathrm{~mm})$, as well as thermal stability for whole system, be better than $1 \mathrm{mK}$. Details of the experimental setup will be described 


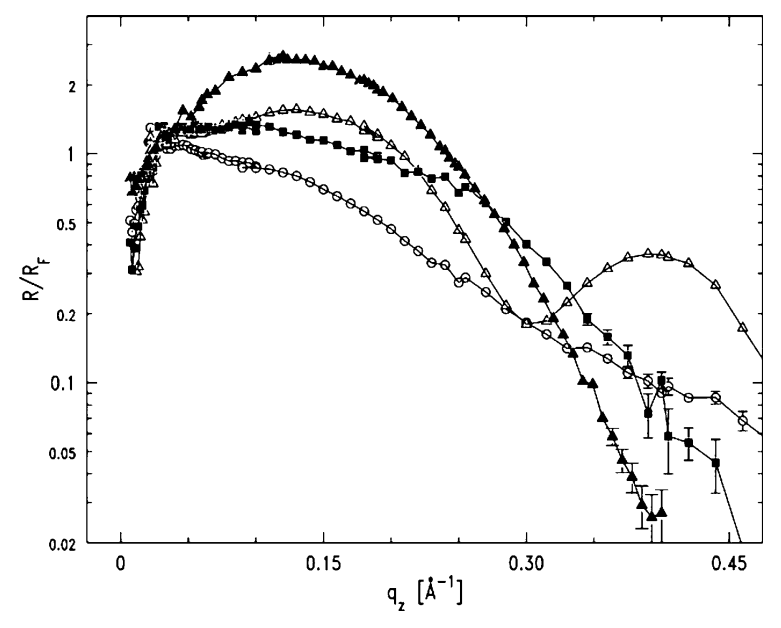

Fig. 2. Normalized X-ray reflectivity $\left(R / R_{\mathrm{F}}\right)$ is shown for the bare molten eicosane surface at $42.2{ }^{\circ} \mathrm{C}$ in the absence of any PFMC in the chamber $(\bigcirc)$, SF phase at $37.2{ }^{\circ} \mathrm{C}(\triangle)$. PFMC was injected in the cell at $37.2^{\circ} \mathrm{C}$ and substrate at $37.2+$ $2.9{ }^{\circ} \mathrm{C} \sim 40.1{ }^{\circ} \mathrm{C}$. Data taken $30 \mathrm{~min}$ after injection into (ם) and in the thermal equilibrium $(\boldsymbol{\Delta})$ illustrate the formation of a wetting layer of PFMC.

elsewhere [13]. The data analysis is based on fitting the measured reflectivity to a theoretical model of the reflected intensity as function of wavevector transfer along the surface normal, $q_{z}=(4 \pi / \lambda) \sin (\alpha)$, where $\lambda$ is the X-ray wavelength and $\alpha$ is the angle of the incident $\mathrm{X}$-ray relative to the surface. The model function used to fit the data is based on the Parratt formalism $[9,12,14]$.

Prior to the wetting measurements X-ray reflectivity was measured from the molten eicosane at the two control points: (i) at $T=37.2^{\circ} \mathrm{C}$, about a degree above eicosane bulk freezing temperature, where SF layer exists, and, (ii) at $T=42.2{ }^{\circ} \mathrm{C}$, where surface does not exhibit SF. In these measurements the chamber was filled with only ultrahigh purity nitrogen. The $q_{z}$ dependence of the $\mathrm{X}$-ray reflectivities, normalized to the Fresnel reflectivity is shown in Fig. 2 for both temperatures. The structureless surface is manifested by the monotonically decaying, normalized curve (open circles) at $42.2{ }^{\circ} \mathrm{C}$, while for the SF monolayer the curve has the clear oscillation signature (open triangles). The results for both temperatures completely agree with those reported by Ocko et al.[9]
A small amount of PFMC, sufficient to provide the reservoir for the vapor $(1.8 \mathrm{ml})$, was injected into the chamber at $T=37.2{ }^{\circ} \mathrm{C}$, while the substrate temperature was kept at a $\Delta T=2.95{ }^{\circ} \mathrm{C}$ above the reservoir. The construction of the chamber eliminated the possibility of macroscopic condensation of the PFMC anywhere except at the sample and the reservoir. The changes in the surface structure were monitored by the X-ray reflectivity at various times following the injection of PFMC.

Data taken 30 min after injection (Fig. 2, solid squares) clearly show the absence of the oscillation signature that is prominent in the SF phase. The fit to the X-ray reflectivity data yields the incomplete PFMC layer with thickness $\sim 7 \AA$. As the system approaches thermal equilibrium this PFMC layer develops into a complete layer of the same thickness. The reflectivity illustrating this is shown by solid triangles in Fig. 2. This unambiguously demonstrates the suppression of the SF phase by the thinnest detected absorbed film of PFMC and supports the view that the existence of SF effects depends on a very fine balance between interfacial energies and inter-chain interactions [9]. However, our experimental result does not rule out another possible explanation of the SF phase. Namely as a phenomenon that is stabilized by the normal fluctuations of alkane chains [15]. Those fluctuations can indeed be considerably suppressed by the presence of the wetting film.

The wetting on C20 by vapor of PFMC was studied at the substrate temperature of $37.2{ }^{\circ} \mathrm{C}$ by varying the $\Delta T$ from 2.95 to $0 \mathrm{~K}$. Examples of the reflectivity curves are shown in Fig. 3. The gradual decrease of the period of oscillations with decreasing $\Delta T$ is due to the growth in thickness of the wetting film. The important feature to note here is the common envelope for all data taken above $\Delta T=0.5 \mathrm{mK}$. This implies the same contrast of the film relative to the molten underlying eicosane bulk, or in other words, the constant electron density of the wetting film. Some divergence from that envelope is observed for the higher momentum transfer $\left(q_{z}\right)$ values, where differences in the film roughnesses contribute more to the X-ray reflectivity. However, note the strong deviation from the common envelope that is ob- 
served at $\Delta T=0.5 \mathrm{mK}$ even for all low $-q_{z}$ data. We attribute this to a combination of the finite angular resolution of the spectrometer and the sample curvature, as well as macroscopic condensation on the C20 substrate due to the vanishing difference between the chemical potentials of the adsorbed liquid and the reservoir.

The analysis indicates film thicknesses as large as 150-300 $\AA$, however unresolved larger thicknesses $(>500 \AA)$ are observed in the range of $\delta(\Delta T)<1 \mathrm{mK}$. This proves the complete wetting scenario and provides an unambiguous basis for setting the empirical zero for $\Delta T$. The true value of the zero in $\Delta T$ is critical for quantitative determination of the power law that governs the dependence of the film thickness $d$ on $\Delta T$. The results of such dependence are shown on a $\log -$ $\log$ scale in Fig. 4. The equilibrium film thickness is defined by the condition, $\partial G / \partial d=0$ and Eq. (1) yields the $d \sim \Delta T^{-1 / 3}$ dependence, when the gravitational effect is negligible. The data support the $1 / 3$ power law for classical wetting and clearly indicate the dominant contribution of van der Waals forces. Note, the largest controllable thickness of the film was about $160 \AA$. In view of the fact that data do not deviate from the $1 / 3$ power law retardation effects must only become important for greater thicknesses.

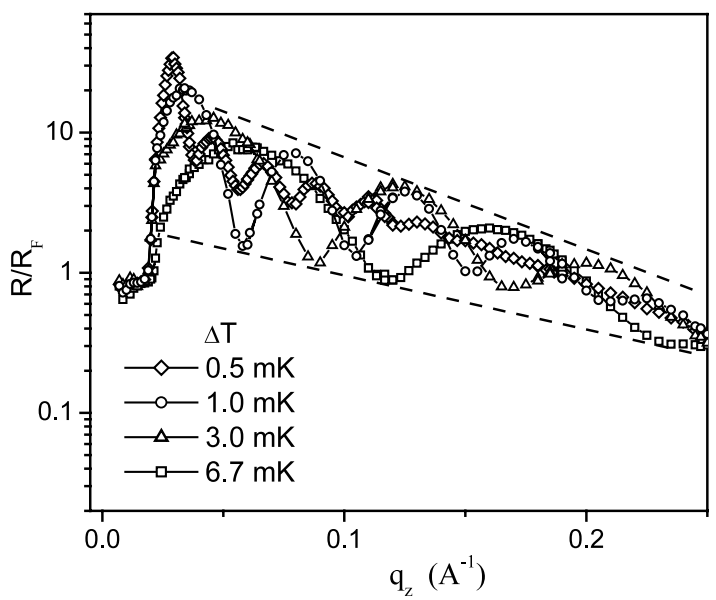

Fig. 3. The normalized reflectivity from the surface of PFMC wetted liquid eicosane for $\Delta T=0.5,1,3,6.7 \mathrm{mK}$. The dashed lines emphasize the common envelope.

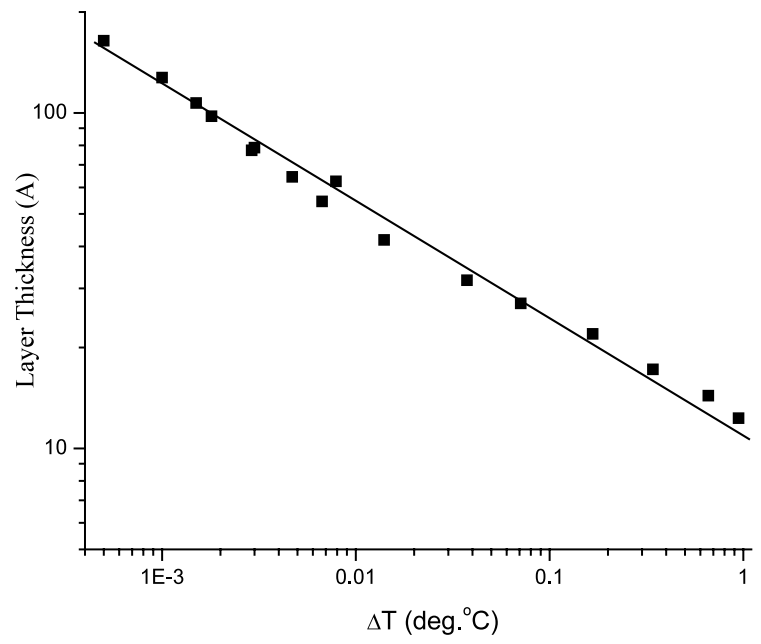

Fig. 4. The film thickness dependence on $\Delta T$ at $T=37.2{ }^{\circ} \mathrm{C}$.

In summary, we have investigated wetting of eicosane, just above its freezing point, by the vapor of PFMC with X-ray reflectivity. We observed that adsorption even thin wetting layer of PFMC is sufficient to completely suppress the SF phase surface phase of eicosane. We made use of controlled temperature offset between the PFMC reservoir and adsorbing substrate to study the dependence of film thickness on $\Delta T$. The observed $1 / 3$ power law indicates retardation effects are negligible for films $\leqslant 160 \AA$.

\section{Acknowledgements}

Work supported by NSF-DMR-98-72817 and Rothschild Foundation. We wish to acknowledge S. Coburn, B. Ocko and E. DiMasi for their technical support at beamline X22B of the NSLS (BNL).

\section{References}

[1] P.G. de Gennes, Rev. Mod. Phys. 57 (1985) 827.

[2] B.M. Law, Prog. Surf. Sci. 66 (2001) 159-216.

[3] M. Schick, in: J. Charvolin, J.F. Joanny, J. Zinn-Justin (Eds.), Liquids at Interfaces, Les Houches Summer School Lectures, vol. XLVIII, North Holland, Amsterdam, 1990, pp. 415-498. 
[4] (a) E. Bertrand, H. Dobbs, D. Broseta, J. Indekeu, D. Bonn, J. Meunier, Phys. Rev. Lett. 85 (2000) 1282-1285; (b) D. Bonn, E. Bertrand, J. Meunier, R. Blossey, Phys. Rev. Lett. 84 (2000) 4661-4664.

[5] T. Pfohl, H. Möhwald, H. Riegler, Langmuir 14 (1998) 5285-5291.

[6] R.K. Heilmann, M. Fukuto, P.S. Pershan, Phys. Rev. B 63 (2001) 205 405-205416.

[7] J.J. Christensen, Handbook of Heats of Mixing, Wiley, New York, 1988.

[8] D.G. LeGrand, G.L. Gaines Jr., J. Coll. Interface Sci. 50 (1975) 272.

[9] B.M. Ocko, X.Z. Wu, E.B. Sirota, S.K. Sinha, O. Gang,
M. Deutsch, Phys. Rev. E 55 (1997) 3164-3182.

[10] B.M. Ocko, E.B. Sirota, M. Deutsch, E. DiMasi, S. Coburn, J. Strzalka, S. Zheng, A. Tronin, T. Gog, C. Venkataraman, Phys. Rev. E 63 (2001) 032602.

[11] I.M. Tidswell, T.A. Rabedeau, P.S. Pershan, S.D. Kosowsky, Phys. Rev. Lett. 66 (1991) 2108.

[12] P.S. Pershan, J. Als-Nielsen, Phys. Rev. Lett. 52 (1984) 759.

[13] O. Gang, M. Fukuto, P. Huber, P.S. Pershan, unpublished.

[14] L.G. Parratt, Phys. Rev. 95 (1954) 359.

[15] A.V. Tkachenko, Y. Rabin, Phys. Rev. Lett. 76 (1996) 2527. 